\title{
EL MOVIMIENTO ANTIVACUNAS: UN ALIADO DE LA COVID-19
}

\section{THE ANTI-VACCINATION MOVEMENT: AN ALLY OF THE COVID-19}

\section{Marta Consuegra-Fernández}

Universitat de Barcelona (UB), Barcelona, España

mconsuegra@ub.edu

Palabras clave: Antivacuna, COVID-19, vacunas, investigación, pandemia.

Keywords: Anti-vaccines, COVID-19, vaccines, research, pandemics.

Resumen. Todo apunta a que el éxito contra la pandemia actual pasa por el desarrollo de una vacuna eficaz que proteja a la población del nuevo coronavirus. Esta protección llegaría a ser global y efectiva si una mayoría de personas recibe la vacuna. No obstante, el creciente movimiento antivacunas podría obstaculizar este efecto rebaño y prolongar la subsistencia del virus entre la población. El presente artículo pretende dar argumentos para un debate necesario sobre el rechazo de la vacunación, ineludible en contexto de emergencia sanitaria por la pandemia originada por SARS-CoV-2.

Abstract. The development of an effective vaccine that protects against the new coronavirus is expected to be the key to overcome the current pandemics. If the vaccine is administered to a majority of the people, this protection could eventually become global. Nevertheless, the growing anti-vaccine movement may hinder the herd effect and lengthen the virus persistence among the population. The present work argues in favor of a necessary debate on vaccination refusal, unavoidable within the framework of health emergency due to the SARS-CoV-2 pandemics.

\section{l. Introducción}

En menos de un año la comunidad científica ha logrado generar un amplio conocimiento sobre el nuevo coronavirus para hacer frente a la pandemia; desde describir su secuencia genómica, es decir, toda la información evolutiva del virus y las instrucciones genéticas para su desarrollo, hasta el mecanismo de penetración en las células humanas, fundamental para el diseño de herramientas que bloqueen la infección. Estos y otros avances científicos han sentado las bases para la investigación de tratamientos efectivos para los enfermos con COVID-19 y vacunas para prevenir futuros contagios. 
Más allá del establecimiento de políticas preventivas como el distanciamiento social, uno de los objetivos que persiguen los científicos actualmente es que la población desarrolle inmunidad frente al SARSCoV-2. Esto es importante porque cuando esta inmunidad alcanza una mayoría de individuos, el 60-70\% (Aguas et al., 2020), la infección se propaga con lentitud por falta de portadores suficientes que permitan extender el virus. Así, las personas protegidas actúan como cortafuegos impidiendo que el patógeno alcance al resto. Este fenómeno se conoce como inmunidad colectiva, de grupo o de rebaño y puede conseguirse o bien a través de la vacunación o bien de forma natural a medida que los individuos van superando la infección y desarrollan anticuerpos contra el virus. No obstante, alcanzar de manera natural esta protección colectiva tiene un coste en vidas elevado. España por ejemplo, acumula ya más de 30.000 fallecidos por COVID-19 y solo el $5 \%$ de la población ha generar anticuerpos contra el virus (Grupo ENE-COVID, 2020). En este caso, lograr el $60 \%$ implicaría más de 300.000 muertes. Por este motivo, los centros de investigación biotecnológicos trabajan en una carrera sin precedentes para conseguir una vacuna que proteja a los individuos y logre una inmunidad suficiente para controlar la pandemia a nivel global. Este hecho ha avivado el activismo antivacuna, especialmente extendido en la última década (Olive et al., 2018) y ahora amenaza con obstaculizar un futuro programa de vacunación contra el nuevo coronavirus.

Esta corriente ha existido desde que se desarrolló la primera vacuna contra la viruela en la década de 1880 y se intensificó particularmente a raíz del controvertido artículo de The Lancet (Wakefield et al.,
1998). En la publicación se establecía una relación causal entre la vacuna triple vírica, contra las enfermedades del sarampión, las paperas y la rubeola, y ciertos comportamientos autistas e incluso inflamaciones intestinales graves. Pese a tratarse de conclusiones absolutamente falsas que escondían conflictos de intereses (Donvan \& Zucker, 2017), se recibieron en la sociedad como sentencias absolutas y se desencadenó una caída considerable del índice de vacunación alrededor de mundo. Desde entonces hasta ahora el movimiento antivacunas persiste y sigue resultando tan peligroso como contagioso, en especial en un contexto de emergencia sanitaria como el actual.

Este escepticismo de la ciencia es un tema ampliamente estudiado y es consecuencia de una extensa diversidad de causas (Rutjens et al., 2018). La orientación política, la moralidad o la cultura científica son algunos de los factores que condicionan la confianza científica. Concretamente, la ideología religiosa, normalmente protestante, se correlaciona con el movimiento antivacunas, mientras que, por ejemplo, la tendencia política no parecería influir (Rutjens et al., 2018). Dada la heterogeneidad de los motivos que dan lugar al descrédito científico, deben implementarse diferentes estrategias para promover la confianza en los distintos aspectos más controvertidos.

Una de las medidas más utilizadas para impulsar la aceptación de las vacunas es la difusión y enseñanza de conocimientos científico-tecnológicos a la población en general, la llamada alfabetización científica o de la salud (Ploomipuu et al., 2019). Esta estrategia devuelve los avances científicos a la sociedad; busca que los descubrimientos y desarrollos tecnológicos sean conocidos, comprendidos y apropiados 
por la sociedad y, en definitiva, que formen parte de la cultura. No obstante, la existencia de movimientos antivacunas en pleno siglo XXI, en poblaciones con sistemas educativos que incluyen educación sobre cuestiones sanitarias, resulta difícil de comprender.

El presente artículo examina y razona los riesgos asociados a las vacunas ya conocidas, y en particular a las candidatas COVID-19, como argumento frente a esta actitud de rechazo. También analiza el origen y las causas de este movimiento popular y evalúa el peligro de un rechazo generalizado, en particular, en un contexto de emergencia sanitaria como el actual. Este conflicto requiere intervenir anticipadamente para concienciar a la población y erradicar cualquier obstáculo que impida un futuro programa de vacunación contra el nuevo coronavirus.

\section{Desarrollo y sequridad de las vacunas COVID-19}

A principios de este año, investigadores del Centro Clínico de Salud Pública de Shanghái publicaron la secuencia genética del SARS-CoV-2, el virus que origina la COVID-19, hecho que desencadenó una ferviente actividad investigadora centrada en el diseño de una vacuna. Actualmente, hay más de cien candidatos contra el nuevo coronavirus en estudio y este número sigue aumentando (Wang et al., 2020). De hecho, hay 36 vacunas que se están probando en humanos actualmente (World Health Organization, 2020)․․ Las principales estrategias para lograrlo buscan generar inmunidad en el huésped

1. Datos actualizados el 19 de septiembre de 2020. humano y abarcan desde la inyección de una proteína vírica o una parte del material genético del virus (ADN o ARN), hasta el propio virus atenuado o inactivado. Tanto en un caso como en otro, en respuesta a la inyección las células del sistema inmunitario generan anticuerpos que reconocen estructuras víricas concretas y que, por tanto, pueden hacer frente a una posible infección futura.

Las vacunas tradicionales, constituidas por virus, son generadas mediante un proceso relativamente sencillo. Estas pueden estar compuestas o bien a partir de virus atenuados o, lo que es lo mismo, menos virulentos, o bien a partir de virus inactivados térmica o químicamente y que, por lo tanto, no están vivos. Las primeras inducen una infección leve que simula la reacción real derivada del patógeno y desencadenan una potente respuesta inmunitaria que el organismo conserva durante años. No obstante, pueden tener complicaciones relativas a su seguridad y originar reacciones adversas en personas con sistemas inmunitarios debilitados (incluye pacientes con enfermedades crónicas, HIV, de edad avanzada o bebés, entre otros) e incluso, en ocasiones, el virus puede revertir su virulencia e infectar a los individuos que reciben la vacuna (Wang et al., 2020). Las vacunas inactivadas son comparativamente más seguras ya que contienen patógenos inertes. Sin embargo, la respuesta inmunitaria que generan no es tan potente y pueden requerir múltiples dosis para generar una protección duradera. Las complicaciones que pueden darse con este tipo de vacunas se asocian, esencialmente, a defectos o errores en el proceso de fabricación.

A diferencia de las dos anteriores, las de nueva generación tienen un mejor perfil de seguridad. Estas no incluyen el virus 
entero, sino que se componen de antígenos o estructuras (también de las secuencias genéticas de ADN o ARN que dan lugar a estos antígenos) que son específicas o únicas del virus. La principal desventaja asociada a este tipo de vacunas es que pueden conferir una baja protección y por lo tanto requieren de otros complementos y más dosis de vacuna para potenciar la eficacia de la respuesta del sistema inmunitario. Para desarrollar este tipo de estrategias es imprescindible acumular un extenso conocimiento de la estructura y de los mecanismos de infección del patógeno por lo que, de entrada, puede implicar más tiempo. En este sentido, la información que pueda tenerse de virus procedentes de la misma familia, como es el caso del coronavirus del Síndrome Respiratorio Agudo Grave (SARS-CoV, de las siglas en inglés) o del Síndrome Respiratorio de Oriente Medio (MERS-CoV), resulta muy útil y puede ayudar a acelerar la identificación de antígenos como posibles vacunas. La proteína conocida como espícula o $S$ (de las siglas en inglés) del SARS-CoV-2 está presente en otros virus de la misma familia y gracias al conocimiento previo se sabe que es crucial en la infección también del SARSCoV-2 ya que le permite al virus acoplarse a la superficie de las células que ataca y, en última instancia, infectarlas. Esta proteína está ubicada en la parte externa de las partículas del virus y desempeña un papel fundamental en el ciclo de vida del virus. Este hecho la erige como una de las posibles dianas terapéuticas más importantes y la mayoría de las candidatas frente al nuevo coronavirus utilizan la proteína S entera o fragmentos de la misma como antígeno (Wang et al., 2020).

Una de las herramientas más modernas que contribuye a acelerar la generación de vacunas incluye el llamado machine learning o deep learning, estrategias de inteligencia artificial, y las técnicas de dinámica molecular (Wang et al., 2020). Estas aproximaciones computacionales permiten simular interacciones con los antígenos y predecir posibles dianas reduciendo así, el tiempo y el coste económico de su desarrollo. Pese a tratarse de enfoques relativamente nuevos, ya se están empleando y se espera que en un futuro puedan ser claves en el diseño de nuevas preparaciones preventivas y terapéuticas.

Uno de los mayores retos de la pandemia de la COVID-19, aparte de la seguridad y efectividad es, sin duda, la producción de la vacuna a gran escala para abastecer las necesidades globales actuales. La demanda es tan elevada que plantea un conflicto para muchos de estos métodos cuya capacidad de producción es limitada. Además, cada procedimiento debe optimizarse para cumplir con los estándares de calidad y equilibrar el coste-volumen de producción; lo que suma tiempo y complejidad. El abastecimiento en regiones alejadas o de difícil acceso también representa un desafío y es que muchas de las vacunas producidas son sensibles a temperaturas altas o tienen una vida media corta.

Por último, la vía de administración puede resultar una complicación añadida y requiere de un proceso de optimización, ya que puede influir en la calidad de las respuestas inmunitarias. En el caso de SARS-CoV-2, los científicos discuten si una vacunación intranasal sería más eficaz que la inyección convencional. Sin embargo la dependencia de un dispositivo de administración especial entre otros inconvenientes (Wang et al., 2020), ha favorecido que las preparaciones en estudio actualmente sean inyectables.

Además de los desafíos aquí citados, la generación de una vacuna contra el nue- 
vo coronavirus debe responder a una demanda masiva y urgencia extrema, por lo que los investigadores buscan acortar el tiempo de obtención de una candidata segura y eficaz.

Para obtener una autorización oficial, estas están sujetas a unas normativas de seguridad particularmente rigurosas y son sometidas a análisis realizados por expertos procedentes de distintas disciplinas como salud pública, epidemiología, inmunología o estadística. En general, su desarrollo puede prolongarse hasta diez (o incluso 20) años para patógenos nuevos o un año para aquellos conocidos, como es el caso del virus de la gripe. Este proceso atraviesa un primer periodo de investigación básica que concluye con experimentos preclínicos en tejidos celulares y modelos animales. Las tres sucesivas fases posteriores (I, II, II) evalúan los efectos biológicos, su seguridad y efectividad en grupos de adultos cada vez más numerosos; de 100 a miles de voluntarios en las etapas finales. El hecho de que las vacunas se administren en personas sanas requiere minimizar las reacciones secundarias que puedan ocasionar. Además de reiterar los estudios de seguridad en cada una de las fases, también se optimizan las dosis y vías de administración. Este proceso culmina en la aprobación de la vacuna y la entrada en la última fase, la IV. En esta etapa se monitorea la eficacia y la aparición de efectos adversos en individuos que se encuentran en un contexto real. Finalmente, las candidatas que han superado todas las pruebas con éxito, tres de cada diez en el mejor de los casos (Wong et al., 2019), se comienzan a distribuir en campañas de vacunación mundial.

El impacto humanitario y económico ocasionado causado por el virus SARS-CoV-2 ha multiplicado los esfuerzos, recursos económicos y herramientas más novedosas para hallar una vacuna a la mayor brevedad. Incluso los expertos sugieren que se podría lograr en menos de 18 meses (Chen et al., 2020)the causative agent of coronavirus disease (COVID-19. De hecho, la primera candidata contra el nuevo coronavirus que se probó en humanos fue el 16 de marzo de $2020^{2}$, pocos meses después del hallazgo del SARS-CoV-2. De hecho, a día de hoy, hay más de una treintena en fase clínica (World Health Organization, 2020); un hecho sin precedentes, con tan poco margen de tiempo.

Esto es posible gracias al esfuerzo global de la comunidad científica apoyada en esta ocasión por una elevada financiación. Además, la naturaleza y características del virus, como, por ejemplo, el hecho de que no tenga tanta facilidad de mutar como el virus de la gripe, también facilitan el proceso. Otro de los elementos que acelera su desarrollo es el hecho de trabajar con vacunas de ARN y ADN, cuya generación pasa por metodologías sintéticas más rápidas debido a que no requieren de cultivo y fermentación. Asimismo, la magnitud de la emergencia justifica el inicio simultáneo de distintas fases clínicas que normalmente seguirían un orden lineal; hecho que por su parte conlleva la asunción de un mayor riesgo financiero (Lurie et al., 2020) En definitiva, dadas estas circunstancias y obtenidos los primeros resultados esperanzadores (Jackson et al., 2020), los expertos confían en poder cumplir con las promesas anunciadas; siempre y cuando se garantice su seguridad. En este sentido, ya se han descrito los primeros efectos adversos ori-

2. BBC News Mundo (16 marzo, 2020). Coronavirus: Estados Unidos comienza a probar la primera vacuna en humanos contra el COVID-19. https:/www.bbc.com/mundo/noticias-51921073 
ginados por algunas vacunas en estudio. Los científicos argumentan que es algo esperable en el proceso de desarrollo y optimización, y que mientras prevalezcan los hallazgos positivos, como hasta ahora, se continuará en la misma línea de investigación (Jackson et al., 2020).

\section{Movimiento anti-vacunas: el caso de la COVID-19}

La historia de la medicina acumular numerosos precedentes de vacunas efectivas como la de la viruela, la poliomielitis - la rabia; casos en los que estas han desempeñado un papel determinante en la reducción del número de enfermos y fallecidos. Estos éxitos han despertado un elevado optimismo respecto a la obtención de la vacuna COVID-19. No obstante, la duda actual es si este optimismo se traduce también en una mayor confianza en su eficacia y si será suficiente para disuadir la opinión más crítica.

Las vacunas han sido víctimas de su propio éxito (Rochel de Camargo, 2020). De acuerdo con la doctora Jenifer Ehreth (Ehreth, 2003), la vacunación salva hasta tres millones de niños cada año y podría llegar a salvar tres más si se generalizara todavía más su uso y se garantizara el acceso en zonas remotas. Se calcula que la erradicación de la viruela, gracias a la vacuna, evitó 350 millones de personas infectadas y 40 millones de muertes por la enfermedad (Ehreth, 2003). Asimismo, el número de casos de poliomielitis notificados desde 1988 se redujo un 99\% y el sarampión, una de las enfermedades más contagiosas y una de las principales causas de muerte infantil en los países en desarrollo (900.000 muertes anuales) disminuyó hasta un 75\% en 1998 (Ehreth,
2003). No solo supone un dramático descenso del número de enfermos y fallecidos, sino que esto también se traduce en un significativo ahorro económico estimado en miles de millones de euros que los centros sanitarios pueden destinar a otros usos. En este sentido, son una de las herramientas de salud pública disponibles más rentables. Para una parte de la población, sin embargo, el beneficio de las vacunas se ha vuelto intangible y se oponen a su uso. Esto tiene un impacto real y medible; los brotes de infecciones por patógenos como el sarampión coinciden con el rechazo de la vacuna por parte de los progenitores que se niegan a administrarla a sus hijos (Benecke \& DeYoung, 2019).

La progresiva desaparición de diversas enfermedades infecciosas graves o incluso letales ha permitido que se subestime la amenaza que suponen y se sobreestimen los riesgos asociados a la vacunación. La realidad es que la prevalencia de efectos adversos derivados de una vacuna en cualquier caso es considerablemente menor. Si evaluamos un ejemplo concreto, como el virus del sarampión (Rochel de Camargo, 2020), para un millón de niños no vacunados infectados, tendríamos 300.000 casos de complicaciones, incluidas 2.000 muertes, mientras que para un millón de niños vacunados niños, se esperarían 34 casos con reacciones adversas importantes, la mayoría de los cuales serían la aparición de trombocitopenia transitoria, con solo un caso de reacción alérgica significativa y menos de un caso de encefalitis.

Pese a la baja prevalencia de efectos secundarios asociados a la vacunación, parte de la opinión pública cuestiona más su seguridad que la propia eficacia, a pesar incluso de ser más seguras que los fármacos terapéuticos tradicionales (WHO, 2008). Esta percepción sesgada tiene 
diversos orígenes. Uno de ellos son los intereses económicos, a menudo ocultos, como condicionantes de las decisiones de los especialistas en salud. Si bien es cierto que los intereses económicos juegan un papel desproporcionado en el sector sanitario, las pérdidas que supondría la comercialización de una vacuna con graves efectos secundarios serían excesivas. En este sentido, el objetivo de las vacunas es prevenir enfermedades, es decir, se administran a personas sanas y por ello es necesario que no causen, como mínimo, más daños de los que podría ocasionar la propia enfermedad. En definitiva, el beneficio de su uso debe ser mayor al riesgo de producir reacciones adversas.

Este descrédito científico además se ve reforzado por estructuras sociocognitivas como el llamado razonamiento motivado. Esto es, una vez una persona tiene una creencia errónea tiende a buscar y considerar más fiables los hechos que argumentan a favor de estas ideas desestimando las evidencias que no se ajustan a su opinión. Otro fenómeno que fortalece las actitudes anti-vacuna es el efecto Dunning-Kruger. Éste describe cómo las personas con menos conocimientos creen saber más o ser más capaces de evaluar información científica que los propios expertos en el campo. Estaría presente en más de un 30\% de la población estudiada (Motta et al., 2018).

Por otro lado, la aversión a la objetividad científica se ha visto afianzado gracias a las nuevas tecnologías de la comunicación. Los argumentos anti-vacunas más presentes en internet se pueden resumir en: (i) la presencia de ingredientes y conservantes tóxicos de las vacunas, sin riesgo real para la salud, (ii) la infraestimación de efectos adversos asociados y reconocidos, generalmente derivado de falacias, anécdotas personales y mal- entendidos, (iii) el cuestionamiento sin fundamento de la profesionalidad de los investigadores que las desarrollan, alimentado por el caso de Wakefield, (iv) la negación de los beneficios obvios derivados de la vacunas erróneamente asociados a mejoras en la higiene general, (v) la existencia cada vez de más vacunas aparentemente innecesarias debido a una desinformación general, (vi) la creencia de que la inmunidad natural es mejor, subestimando los riesgos de exposición a enfermedades prevenibles y (vii) la intuición de los progenitores que debería prevalecer sobre cualquier evidencia científica (Rochel de Camargo, 2020)La mayoría de las argumentaciones que contradicen la vacunación son infundadas y no tienen un respaldo riguroso ni evidencias fiables, pero el acceso ilimitado a la información junto con el uso masivo de las redes sociales han alimentado la masa crítica y han facilitado la creación de comunidades anticientíficas cada vez más numerosas (Benecke \& DeYoung, 2019).

Si bien es cierto que el desarrollo de vacunas no ha estado exento de errores como el suceso de la vacuna Salk contra la poliomielitis (Offit, 2005), el progreso tecnológico y los avances científico-médicos han contribuido enormemente a su seguridad y eficacia. En este sentido, la comunidad científica rechaza aquellas candidatas que no hayan alcanzado los niveles de calidad exigidas. Este el caso del ensayo de la vacuna COVID-19 rusa, cuyos resultados podrían haber sido manipulados (Abbott, 2020) o las vacunas caseras contra el coronavirus ${ }^{3}$.

3. The New York Times (1 septiembre, 2020). DIY Coronavirus Vaccines? These Scientists Are Giving Themselves Their Own. https://www. nytimes.com/2020/09/01/science/covid-19-vaccine-diy.html 
En el contexto de la pandemia actual, origen natural del SARS-CoV-2, la rápida diseminación del virus o incluso los intereses ocultos de la vacuna se han asociados a conspiraciones diversas. Estas especulaciones proceden del descrédito en la medicina que hoy en día supera el 30\% en algunos territorios (Gallup, 2019).

A pesar de que el apoyo general a las vacunas sigue siendo alto y que desconocemos cuántas personas se opondrían a la vacuna COVID-19 una vez comercializada, los resultados del estudio COSMOSpain impulsado por la Organización Mundial de la Salud (OMS) apuntan que el 30\% de los españoles se negarían a administrársela (Instituto de Salud Carlos III, 2020).

Este rechazo, aunque minoritario, podría llegar a ser devastador en el escenario de emergencia actual; no solamente por la exposición individual a un virus cuya enfermedad no tiene tratamiento, sino por el impedimento que supondría alcanzar la inmunidad colectiva (Ball, 2020; Khan et al., 2020).

\section{Consideraciones finales}

Más allá del aislamiento social, los equipos de protección personal y las medidas de higiene, actualmente la vacunación parece ser la única forma de proteger a la población y poner fin a la pandemia de la COVID-19. La inmunidad colectiva que hemos alcanzado de forma natural es muy variable entre territorios (Britton et al., 2020) y está lejos de lograr el umbral requerido para ser efectiva. El desarrollo de una vacuna COVID-19 es difícil y complejo, como lo es su fabricación masiva y distribución global. No obstante, el conocimiento que la comunidad científica ha acumulado acerca de la naturaleza del virus junto con los avances tecnológicos de los últimos años hace posible pensar que dispondremos de una vacuna segura y eficaz en un periodo de tiempo relativamente breve y, cuando esto sea una realidad, es imprescindible que al menos se le administre a la mitad de la población. Garantizar este porcentaje a nivel mundial implica un compromiso y responsabilidad tanto individual como colectivo y es preciso anticipar aquellos factores que puedan obstaculizar este propósito. El movimiento popular antivacunas existe desde 1880 y, pese a la amenaza que supone, los intentos por detenerlo no han conseguido evitar que en las últimas décadas haya crecido notablemente (Olive et al., 2018). El escenario de emergencia sanitaria actual demanda una respuesta anticipada y cooperativa que disuada la percepción negativa de las vacunas. De otra manera, las implicaciones que tendría un rechazo de la vacuna COVID-19 podrían dinamitar los esfuerzos y recursos que se están dedicando actualmente y retrasar el desarrollo de la inmunidad colectiva.

A diferencia de países como Italia o Francia (Warren, 2019), la vacunación en España es voluntaria, ya que nuestro ordenamiento no incorpora explícitamente el deber de vacunación y nadie puede, en principio, ser obligado a vacunarse. Ahora bien, hay determinadas situaciones que permiten que los poderes públicos competentes impongan la vacunación forzosa, fundamentalmente en caso de epidemias (Comité Asesor de Vacunas de la AEP, 2018). Se trata de una medida paternalista que atenta las libertades individuales y podría generar rechazo entre la población, como ha sucedido ya en algunos territorios (Valera et al., 2019). En este sentido, 
el sistema británico parece haber solucionado la desconfianza en las vacunas sin recurrir a la imposición (Offit \& Salisbury, 2012) y además, los controvertidos hallazgos procedentes de distintos estudios (ASSET Reports, 2013; Vaz et al., 2020) no aseguran que la implementación de medidas coercitivas pueda aumentar la tasa de vacunación en Europa.

La excepcionalidad de la situación actual podría justificar la obligatoriedad de la vacunación para prevenir la COVID-19 en el caso de disponer de una vacuna y detectar una baja tasa de vacunación. No obstante, existen otras actuaciones que pueden prevenir anticipadamente este escenario sin tener que recurrir a una imposición legal.

Las causas del movimiento antivacunas son múltiples y de naturaleza muy diversa, por lo que parece improbable que puedan abordarse con una única aproximación. En este sentido, las campañas basadas exclusivamente en la educación cambian mínimamente las tasas de vacunación (Miembros del Comité de Bioética de España, 2016) hecho que apoyaría la implementación de diferentes iniciativas combinadas. Así, parece esencial investigar la procedencia multifactorial de la desconfianza frente a las vacunas e idear un plan coordinado que incluya distintas actuaciones como la alfabetización en términos de salud, una comunicación eficiente, la transmisión de información rigurosa y clara, la transparencia de los avances clínicos, la participación de la comunidad científica en la difusión del conocimiento y la promoción de conductas favorables por parte de la población.

A pesar de tratarse de un movimiento popular minoritario, la corriente anti-vacunas tiene un gran potencial para per- suadir a las audiencias más indecisas y seguir creciendo los próximos años (Burki, 2020). De no actuar con anticipación, cualquier futura vacuna COVID-19 podría ser rechazada, en cuyo caso sería necesario recurrir a medidas coercitivas para garantizar la eficacia de la vacunación y la protección de la población frente al virus.

\section{Bibliografía}

Abbott, A. (2020). Researchers highlight 'questionable' data in Russian coronavirus vaccine trial results. Nature, 585(7826), 493-493. https://doi. org/10.1038/d41586-020-02619-4

Aguas, R., Corder, R. M., King, J. G., Gonçalves, G., Ferreira, M. U., \& Gabriela Gomes, M. M. (2020). Herd immunity thresholds for SARS-CoV-2 estimated from 1 unfolding epidemics. medRxiv, 2020.07.23.20160762. https:// doi.org/10.1101/2020.07.23.20160762

ASSET. (2017). Compulsory vaccination and rates of coverage immunistion in Europe, 1-6. http://www.asset-scienceinsociety.eu/reports/page1.html

Ball, P. (2020). Anti-vaccine movement could undermine efforts to end coronavirus pandemic, researchers warn. Nature, 581(7808), 251. https://doi.org/10.1038/ d41586-020-01423-4

Benecke, O., \& DeYoung, S. E. (2019). AntiVaccine Decision-Making and Measles Resurgence in the United States. Global Pediatric Health, 6, 23333794X1986294. https:// doi.org/10.1177/2333794x19862949

Britton, T., Ball, F., \& Trapman, P. (2020). A mathematical model reveals the influence of population heterogeneity on herd immunity to SARS-CoV-2. Science, 369(6505), 846-849. https://doi.org/10.1126/science. abc6810 
Burki, T. (2020). The online anti-vaccine movement in the age of COVID-19. The Lancet Digital Health, 2(10), e504e505. https://doi.org/10.1016/s25897500(20)30227-2

Chen, W. H., Strych, U., Hotez, P. J., \& Bottazzi, M. E. (2020). The SARS-CoV-2 Vaccine Pipeline: an Overview. Current Tropical Medicine Reports, 7(2), 61-64. https://doi.org/10.1007/s40475-02000201-6

Comité Asesor de Vacunas de la AEP. (2018, octubre). Capítulo 44. Manual de vacunas en línea de la Asociación Española de Pediatría (AEP). https://vacunasaep.org/printpdf/documentos/manual/ cap-44

Donvan, J., \& Zucker, C. (2017). Outra Sintonia. A História do Autismo. Companhia Das Letras.

Ehreth, J. (2003). The global value of vaccination. Vaccine, 21(7-8), 596600. https://doi.org/10.1016/S0264410X(02)00623-0

GALLUP. (2019). Wellcome Global Monitor-First Wave Findings. 1-132. https:// wellcome.org/sites/default/files/wellcomeglobal-monitor-2018.pdf

Grupo ENE-COVID. (2020). Estudio ENECOVID: Informe final estudio nacional de sero-epidemiología de la infección por Sars-CoV-2 en España (1-32). Extraído de http://www.thelancet.com/journals/lancet/ article/PIIS0140-6736

Instituto de Salud Carlos III. (2020, julio). Monitorización del comportamiento y las actitudes de la población relacionadas con el COVID-19 en España (COSMOSPAIN): Estudio OMS. COSMO-SPAIN. https://portalcne.isciii.es/cosmo-spain/

Jackson, L. A., Anderson, E. J., Rouphael, N. G., Roberts, P. C., Makhene, M., Coler, R. N., McCullough, M. P., Chappell, J. D., Denison, M. R., Stevens, L. J., Pruijss- ers, A. J., McDermott, A., Flach, B., DoriaRose, N. A., Corbett, K. S., Morabito, K. M., O'Dell, S., Schmidt, S. D., Swanson, P. A., ... Beigel, J. H. (2020). An mRNA Vaccine against SARS-CoV-2 — Preliminary Report. New England Journal of Medicine. https://doi.org/10.1056/nejmoa2022483

Khan, Y. H., Mallhi, T. H., Alotaibi, N. H., Alzarea, A. I., Alanazi, A. S., Tanveer, N., \& Hashmi, F. K. (2020). Threat of COVID-19 vaccine hesitancy in Pakistan: The need for measures to neutralize misleading narratives. American Journal of Tropical Medicine and Hygiene, 103(2), 603-604. https://doi.org/10.4269/ajtmh.20-0654

Lurie, N., Saville, M., Hatchett, R., \& Halton, J. (2020). Developing Covid-19 Vaccines at Pandemic Speed. New England Journal of Medicine, 382(21), 1969-1973. https://doi.org/10.1056/NEJMp2005630

Miembros del Comité de Bioética de España. (2016). Cuestiones ético-legales del rechazo a las vacunas y propuestas para un debate necesario .

Motta, M., Callaghan, T., \& Sylvester, S. (2018). Knowing less but presuming more: Dunning-Kruger effects and the endorsement of anti-vaccine policy attitudes. Social Science and Medicine, 211, 274-281. https://doi.org/10.1016/j. socscimed.2018.06.032

Offit, P. A. (2005). The cutter incident, 50 years later. New England Journal of Medicine, 352(14), 1411-1412. https:// doi.org/10.1056/NEJMp048180

Offit, P. A., \& Salisbury, D. M. (2012). Should childhood vaccination be mandatory? Yes/No. BMJ (Online), 344(7858). https://doi.org/10.1136/bmj.e2435

Olive, J. K., Hotez, P. J., Damania, A., \& Nolan, M. S. (2018). The state of the antivaccine movement in the United States: A focused examination of nonmedical exemptions in states and counties. PLOS 
Medicine, 15(6), e1002578. https://doi. org/10.1371/journal.pmed.1002578

Ploomipuu, I., Holbrook, J., \& Rannikmäe, M. (2019). Modelling health literacy on conceptualizations of scientific literacy. Health Promotion International. https:// doi.org/10.1093/heapro/daz106

Rochel de Camargo, K. (2020). Here we go again: the reemergence of anti-vaccine activism on the Internet. Cadernos de saude publica, 36 2, e00037620. https:// doi.org/10.1590/0102-311x00037620

Rutjens, B. T., Sutton, R. M., \& van der Lee, R. (2018). Not All Skepticism Is Equal: Exploring the Ideological Antecedents of Science Acceptance and Rejection. Personality and Social Psychology Bulletin, 44(3), 384-405. https://doi. org/10.1177/0146167217741314

Valera, L., Vergara, P. R., Barreaux, I. P., \& García, P. B. (2019). Parental refusal to mandatory vaccination in Chile. Ethical and legal challenges. Revista Chilena de Pediatria, 90(6), 675-682. https://doi. org/10.32641/rchped.v90i6.1002

Vaz, O. M., Ellingson, M. K., Weiss, P., Jenness, S. M., Bardají, A., Bednarczyk, R. A., \& Omer, S. B. (2020). Mandatory vaccination in Europe. Pediatrics, 145(2). https://doi.org/10.1542/peds.2019-0620

Wakefield, A. J., Murch, S. H., Anthony, A., Linnell, J., Casson, D. M., Malik, M., Berelowitz, M., Dhillon, A. P., Thomson, M. A., Harvey, P., Valentine, A., Davies, S. E., \& Walker-Smith, J. A. (1998). Retracted: Ileal-lymphoid-nodular hyperplasia, non-specific colitis, and pervasive developmental disorder in children. Lancet, 351(9103), 637-641. https://doi. org/10.1016/S0140-6736(97)11096-0

Wang, J., Peng, Y., Xu, H., Cui, Z., \& Williams, R. O. (2020). The COVID-19 Vaccine Race: Challenges and Opportunities in Vaccine Formulation. AAPS Pharm-
SciTech, 21(6). https://doi.org/10.1208/ s12249-020-01744-7

Warren, M. (2019). Vaccination rates rise in Italy and France after law change. Nature. https://doi.org/10.1038/d41586019-02193-4

WHO. (2008). Vaccination greatly reduces disease, disability, death and inequity worldwide. Bulletin of the World Health Organization, 86(2).

Wong, C. H., Siah, K. W., \& Lo, A. W. (2019). Estimation of clinical trial success rates and related parameters. Biostatistics, 20(2), 273-286. https://doi. org/10.1093/biostatistics/kxx069

World Health Organization. (2020).

Draft landscape of COVID-19 candidate vaccines. https://www.who.int/ publications/m/item/draft-landscape-ofcovid-19-candidate-vaccines 
